\title{
Torsion-free abelian semigroup rings IX
}

\author{
RYÛKI MATSUDA*
}

Let $S$ be a torsion-free cancellative abelian semigroup $\ni 0$ ( nonzero and written additively). Let $G$ be the quotient group of $S$. In $\S 1$, we give a very simple proof of a generalization of [12, Theorem 4]. In $\S 2$, first we prove semigroup versions of $\S 1$ and next we apply them for semigroup rings. In $\S 3$, we prove Prüfer v-multiplication versions of $\S 2$. In $\S 4$, we show that [1], [3], [6] and [7] hold for $S$.

Conjecture. Almost all multiplicative ideal theory for an integral domain holds for $S$.

Not a few examples and $\S 4$ show that the above conjecture seems to be true. Often the proofs of them are almost trivial analogy of these for domains. However they are of use for instanse for semigroup rings. As to this report confer (2. 9) and (3. 10). Moreover, since the ideal theory of $S$ is simpler than that of an integral domain $D$, we may have the means of developing the ideal theory of $S$ for us to develope it of $D$.

§1. We studied a characterization of v-domains in [12]. Let $\mathrm{F}(R)$ be the set of nonzero fractional ideals of $R$, and let $\mathrm{f}(R)$ be the set of finitely generated members of $\mathrm{F}(R)$. If, for each regular element $f$ of $R[X]$, the content ideal of $f$ is a regular ideal of $R$, then $R$ is said to have property (C). If each regular ideal of $R$ is generated by regular elements of $R$, then $R$ is called Marot ring. We proved the following,

(1. 1) ([12, TheOREM 4]). Let * be an e.a.b. star-operation on a Marot ring $R$ with property $(C)$. Assume that $\left(A A^{-1}\right)^{*}=R$ for each regular $A \in f(R)$. Then we have

for all regular $A_{i} \in f(R)$.

$$
\left(A_{1} \cap \cdots \cap A_{n}\right)^{*}=\left(A_{1}\right)^{*} \cap \cdots \cap\left(A_{n}\right)^{*}
$$

(1. 2) ([12, TheOREM 6]). Let $R$ be a Marot ring with property (C). Then $R$ is a $v$-ring if and only if $R$ is integrally closed and

$\left(A_{1} \cap \cdots \cap A_{n}\right)^{v}=($
for all regular $A_{i} \in \mathcal{R}(R)$.

Received January 17, 1994.

1991 Mathematics Subject Classification. Primary 13A02 Secondary 20M25.

* Department of Mathematics, Ibaraki University, Mito, Ibaraki 310, Japan. 
(1. 3) ([12, CorollaRY 7]). Let $D$ be a domain. Then $D$ is a v-domain if and only if $D$ is integrally closed and

for all $A_{i} \in f(D)$.

$$
\left(A_{1} \cap \cdots \cap A_{n}\right)^{v}=\left(A_{1}\right)^{v} \cap \cdots \cap\left(A_{n}\right)^{v}
$$

(1. 3) is the answer to the question of $[3$, p. 7].

Concerning (1. 1), we remark that, let $R$ be a Marot ring with property (C). Assume that $R$ is a Prüfer $*$-multiplication ring, and hence $\left(A A^{-1}\right)^{*}=R$ for each regular $A \in \mathrm{f}(R)$. Then $*$ need not be e.a.b. ([10, Remark 7]).

We are able to generalize (1. 1) as follows.

(1. 4) THEOREM. Let * be a star-operation on a Marot ring $R$. Assume that $\left(A A^{-1}\right)^{*}=R$ for each regular $A \in f(R)$. Then we have

$\left(A_{1} \cap \cdots \cap A_{n}\right)^{*}=\left(A_{1}\right)^{*} \cap \cdots \cap\left(A_{n}\right)^{*}$

for each regular $A_{i} \in f(R)$.

PROOF. We have

$\left(\left(A_{1}\right)^{*} \cap \cdots \cap\left(A_{n}\right)^{*}\right)\left(A_{2} \cdots A_{n}, \cdots \cdots, A_{1} \cdots A_{n-1}\right)^{*} \subset\left(A_{1} \cdots A_{n}\right)^{*}$.

Multiplying $\left(A_{2} \cdots A_{n}, \cdots \cdots, A_{1} \cdots A_{n-1}\right)^{-1}$ for the both sides, we have

$\left(A_{1}\right)^{*} \cap \cdots \cap\left(A_{n}\right)^{*} \subset\left(A_{1} \cap \cdots \cap A_{n}\right)^{*}$.

We stated in [12] as follows, "We may perhaps be able to prove it without using the Kronecker function ring." The above proof of (1.4) is simpler than that of [12, Theorem 4] and does not use Kronecker function rings.

The similar proof with (1. 4) implies the following,

(1. 5). Let * be a star-operation on a Marot ring $R$. Assume that $\left(A A^{-1}\right)^{*}=R$ for each regular $A \in F(R)$. Then we have

$\left(A_{1} \cap \cdots \cap A_{n}\right)^{*}=\left(A_{1}\right)^{*} \cap \cdots \cap\left(A_{n}\right)^{*}$

for each regular $A_{i} \in F(R)$.

$[1$, p.2550] asks to character the integrally closed domain $D$ for which $\left(A_{1} \cap A_{2}\right)^{v}=\left(A_{1}\right)^{v} \cap\left(A_{2}\right)^{v}$ for each $A_{i} \in \mathrm{F}(D)$.

We note that [10, Theorem 5] holds for a Marot ring without property (C). (1. 4) implies the following generalization of (1. 2).

(1. 6) PROPOSITION. Let $R$ be a Marot ring. Then $R$ is a v-ring if and only if $R$ is integrally closed and

$\left(A_{1} \cap \cdots \cap A_{n}\right)^{v}=\left(A_{1}\right)^{v} \cap \cdots \cap\left(A_{n}\right)^{v}$

for each regular $A_{i} \in f(R)$.

§2. The aim of this section is to prove semigroup versions of the results of $\S 1$ and apply them for semigroup rings. We refer [11, Section 10] and [13] for star-operations on semigroups.

Set $\mathrm{q}(S)=G$. Let $\mathrm{F}(S)$ be the set of fractional ideals of $S$, and $\mathrm{f}(S)$ be the set of finitely generated fractional ideals of $S$. 
(2. 1). If $S$ is essential, then for all $A_{i} \in f(S)$.

$\left(A_{1} \cap \cdots \cap A_{n}\right)^{v}=\left(A_{1}\right)^{v} \cap \cdots \cap\left(A_{n}\right)^{v}$

The proof is similar with that of $[3$, Theorem 7 (1)].

(2. 2) PROPOSITION. Let * be a star-operation on $S$. Assume that $\left(A+A^{-1}\right)^{*}=S$ for each $A \in f(S)$. Then we have

$\left(A_{1} \cap \cdots \cap A_{n}\right)^{*}=\left(A_{1}\right)^{*} \cap \cdots \cap\left(A_{n}\right)^{*}$

for all $A_{i} \in f(S)$.

PROOF. The straightforward semigroup version of that of (1. 4). (cf. [13]).

We note that we have the notion of Kronecker function ring of semigroups

Similarly we have

(2. 3). Let * be a star-operation on $S$. Assume that $\left(A+A^{-1}\right)^{*}=S$ for each $A \in F(S)$. Then we have

$\left(A_{1} \cap \cdots \cap A_{n}\right)^{*}=\left(A_{1}\right)^{*} \cap \cdots \cap\left(A_{n}\right)^{*}$

for each $A_{i} \in F(S)$.

As a corollary to (2. 2), we have

(2. 4) COROLLARY. Let * be a star-operation on $S$ of finite character. Assume that $\left(A+A^{-1}\right)^{*}=S$ for each $A \in f(S)$. Then we have

$\left(A_{1} \cap \cdots \cap A_{n}\right)^{*}=\left(A_{1}\right)^{*} \cap \cdots \cap\left(A_{n}\right)^{*}$ for each $A_{i} \in F(S)$.

We give a necessary and sufficient condition for

$\left(A_{1} \cap \cdots \cap A_{n}\right)^{*}=\left(A_{1}\right)^{*} \cap \cdots \cap\left(A_{n}\right)^{*}$

for each $A_{i} \in \mathrm{F}(S)$ for a finite character star-operation $*$ on $\mathrm{S}$ in (3. 11).

(2. 5). (1) If $S$ is integrally closed, then we have $A: A=S$ for each $A \in f(S)$.

(2) We have $A^{v}=\cap\{(\alpha) \mid \alpha \in G$ with $(\alpha) \supset A\}$ for each $A \in F(S)$.

(2. 6). The following conditions are equivalent for $S$.

(1) The v-operation on $S$ is e.a.b.

(2) $A^{v}: A^{v}=S$ for each $A \in f(S)$.

(3) $\left(A+A^{-1}\right)^{v}=S$ for each $A \in f(S)$.

If the v-operation on $S$ is e.a.b., then $S$ is called v-semigroup or regularly integrally closed semigroup. 
(2. 7) COROLLARY. $S$ is a v-semigroup if and only if $S$ is integrally closed and

$$
\left(A_{1} \cap \cdots \cap A_{n}\right)^{v}=\left(A_{1}\right)^{v} \cap \cdots \cap\left(A_{n}\right)^{v}
$$

for all $A_{i} \in f(S)$.

PROOF. The necessity follows by (2. 2) and (2. 6). The sufficiency: Let $A \in \mathrm{f}(S)$. We choose elements $\alpha_{i}$ such that $A=\left(\alpha_{1}, \cdots, \alpha_{n}\right)$. Now, let $(\alpha)$ be a principal fractional ideal of $S$ containing $A+A^{-1}$. Then $-\alpha+A \subset A^{v}$, so that

$$
\begin{aligned}
-\alpha \in A^{v}: A & =\bigcap_{1}^{n}\left(A^{v}:\left(\alpha_{i}\right)\right)=\bigcap_{1}^{n}\left(-\alpha_{i}+A^{v}\right)=\left(\bigcap_{1}^{n}\left(-\alpha_{i}+A\right)\right)^{v} \\
& =(A: A)^{v}=S .
\end{aligned}
$$

Therefore $S \subset(\alpha)$, hence $\left(A+A^{-1}\right)^{v}=S$. By (2. 6), $S$ is a v-semigroup.

(2. 8) ([11, TheOREM (11. 4) (2)]). $D[X ; S]$ is a v-domain if and only if $D$ is a v-domain and $S$ is a $v$-semigroup.

(1. 3), (2. 7) and (2.8) imply the following,

(2. 9) THEOREM. Assume that $D$ is integrally closed and $S$ is integrally closed. Then the following conditions are equivalent.

$$
\text { (1) }\left(A_{1} \cap \cdots \cap A_{n}\right)^{v}=\left(A_{1}\right)^{v} \cap \cdots \cap\left(A_{n}\right)^{v}
$$

for each $A_{1}, \cdots, A_{n} \in f(D[X ; S])$.

$$
\text { (2) }\left(A_{1} \cap \cdots \cap A_{n}\right)^{v}=\left(A_{1}\right)^{v} \cdots\left(A_{n}\right)^{v}
$$

for each $A_{1}, \cdots, A_{n} \in f(D)$ and

$$
\left(A_{1} \cap \cdots \cap A_{n}\right)^{v}=\left(A_{1}\right)^{v} \cap \cdots \cap\left(A_{n}\right)^{v}
$$

for each $A_{1}, \cdots, A_{n} \in f(S)$.

The second proof of the necessity of (2. 7). Let $k$ be a field. By (2. 8), $k[X ; S]$ is a v-domain. Let $A_{1}, \cdots, A_{n} \in \mathrm{f}(S)$. By (1. 3), we have

$$
\left(A_{1} k[X ; S] \cap \cdots \cap A_{n} k[X ; S]\right)^{v}=\left(A_{1} k[X ; S]\right)^{v} \cap \cdots \cap\left(A_{n} k[X ; S]\right)^{v} \text {. }
$$

Let $\alpha \in\left(A_{1}\right)^{v} \cap \cdots \cap\left(A_{n}\right)^{v}$. Then $\alpha \in\left(A_{i} k[X ; S]\right)^{v}$ for each $i$. Hence we have

$\alpha \in\left(A_{1} k[X ; S] \cap \cdots \cap A_{n} k[X ; S]\right)^{v}=\left(\left(A_{1} \cap \cdots \cap A_{n}\right) k[X ; S]\right)^{v}$. It follows $\alpha \in\left(A_{1} \cap \cdots \cap A_{n}\right)^{v}$, and the proof is complete.

$\S 3 . \quad$ Now we study Prüfer v-multiplication version of $\S 2$. For that purpose, we will study semigroup versions of Griffin([6]) first.

Let $\Gamma$ be a totally ordered abelian group. If a nonempty subset $U \underset{\neq}{\subset} \Gamma$ satisfy the following condition, $U$ is called an upper class of $\Gamma$ : $U$ contains each $\beta \in \Gamma$ which is greater than some element of $U$. We may define a natural addition 
on the set $\Lambda$ of upper classses of $\Gamma$. $\Lambda$ becomes a totally ordered semigroup: $U \geq U^{\prime}$ if and only if $U \subset U^{\prime}$. Let $w$ be a valuation on $G$ nonnegative on $S$. If for each $\alpha>0$ there exists $s \in S$ such that $w(s)=\alpha$, then $w$ is called well-centered on $S$. Let $\Omega$ be a family of well-centered valuations on $G$ such that $\bigcap_{\Omega} V_{w}=S\left(V_{w}\right.$ is the valuation semigroup of $w$ ). Let $\Gamma_{w}$ be the value group of $w$, and $\Lambda_{w}$ the upper classes of $V_{w}$. We make a semigroup $\Pi_{\Omega} \Lambda_{w}$ and introduce the componentwise order. For $M \in \mathrm{F}(S)$, we denote the element $(\cdots, w(M), \cdots) \in \Pi \Lambda_{w}$ by $\Phi(M)$.

(3. 1).

(1) $M \subset N$ implies $\Phi(M) \geq \Phi(N)$.

(2) $\Phi(M+N)=\Phi(M)+\Phi(N)$.

(3) $\Phi((M, N))=\inf (\Phi(M), \Phi(N))$.

(4) $\Phi\left(\left(x_{1}, \cdots, x_{n}\right)+M\right)=\Phi\left(\left(x_{1}, \cdots, x_{n}\right)+N\right)$ implies $\Phi(M)=\Phi(N)$.

If all the valuations of $\Omega$ are essential, we have in addition:

(5) $\Phi(M \cap N)=\sup (\Phi(M), \Phi(N))$.

(6) $\Phi(M+(L \cap N))=\Phi((M+L) \cap(M+N))$.

(7) $\Phi((M, N)+(M \cap N))=\Phi(M+N)$.

(8) $\Phi(M \cap(L, N))=\Phi((M \cap L, M \cap N))$.

For $M \in \mathrm{F}(S)$, we set $M^{c}=\cup\{N \in \mathrm{F}(S) \mid \Phi(N)=\Phi(M)\}$. The mapping $M \longmapsto M^{c}$ becomes a star-operation on $S$.

(3. 2). We have $M^{c}=\bigcap_{\Omega}\left(M+V_{w}\right)$.

(3. 3). The following conditions are equivalent.

(1) For each $M \in f(S)$, we have $\left(M+M^{-1}\right)^{v}=S$.

(1') For each $M \in f(S)$ and $N_{1}, N_{2} \in F(S)$, we have

$\left(M^{v}+\left(N_{1}^{v} \cap N_{2}^{v}\right)\right)^{v}=\left(M^{v}+N_{1}^{v}\right)^{v} \cap\left(M^{v}+N_{2}^{v}\right)^{v}$.

(2) There exists a star-operation * on $S$ such that for each $M \in f(S)$ and $N_{1}, N_{2} \in F(S)$, we have

$\left(M^{*}+\left(N_{1}^{*} \cap N_{2}^{*}\right)\right)^{*}=\left(M^{*}+N_{1}^{*}\right)^{*} \cap\left(M^{*}+N_{2}^{*}\right)^{*}$.

(3) There exists a star-operation * on $S$ such that for each $M, N \in f(S)$, we have

$\left(\left(M^{*}, N^{*}\right)+\left(M^{*} \cap N^{*}\right)\right)^{*}=\left(M^{*}+N^{*}\right)^{*}$

Moreover, (2) implies (3) for the same star-operation *. (3) implies that for each $M \in f(S)$ we have $\left(M+M^{-1}\right)^{*}=S$.

(3. 4). Let * be a star-operation of finite character on $S$. Let $\Pi$ be the set of maximal *-ideals of $S$. Then for each $M \in F(S)$ we have,

(1) $M^{*}=\bigcap_{\Pi}\left(M^{*}+S_{P}\right)$.

(2) If $M^{*}=S$, then $S=\bigcap_{\Pi}\left(M+S_{P}\right)$ and $S_{P}=M+S_{P}$.

(3. 1) (3. 4) imply the following, 
(3. 5) PROPOSITION. Let * be a star-operation of finite character on $S$. Then the following conditions are equivalent.

(1) $S$ is a Prüfer *-multiplication semigroup.

(2) For each maximal *-ideal $P, S_{P}$ is a valuation semigroup.

(3) For each $L, M, N \in F(S)$, we have

$\left(L^{*}+\left(M^{*} \cap N^{*}\right)\right)^{*}=\left(L^{*}+M^{*}\right)^{*} \cap\left(L^{*}+N^{*}\right)^{*}$.

(4) For each $M, N \in F(S)$, we have

$\left(\left(M^{*}, N^{*}\right)+\left(M^{*} \cap N^{*}\right)\right)^{*}=\left(M^{*}+N^{*}\right)^{*}$.

(5) For each $M \in f(S)$, we have $\left(M+M^{-1}\right)^{*}=S$.

(6) For each $L, M, N \in F(S)$, we have

$\left(L^{*} \cap\left(M^{*}, N^{*}\right)\right)^{*}=\left(L^{*} \cap M^{*}, L^{*} \cap N^{*}\right)^{*}$ $M \in F(S)$.

Moreover these conditions imply that $M^{c}=M^{*}=\bigcap_{\Pi}\left(M+S_{P}\right)$ for each

Concerning (2. 3), we see the following. Let $*$ be a finite character staroperation on $S$. Assume that

$$
\left(A_{1} \cap \cdots \cap A_{n}\right)^{*}=\left(A_{1}\right)^{*} \cap \cdots \cap\left(A_{n}\right)^{*}
$$

for each $A_{i} \in \mathrm{F}(S)$. Then $\left(A+A^{-1}\right)^{*}=S$ need not hold for each $A \in \mathrm{f}(S)$.

A counter example: Assume that $S$ is not a valuation semigroup. Let * be the identity mapping of $\mathrm{F}(S)$. Then, by (3. 5) there exist $A \in \mathrm{f}(S)$ such that

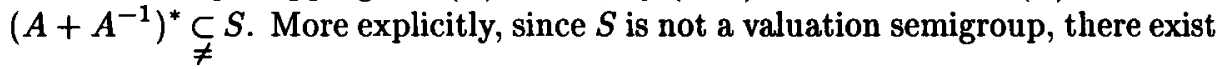
$s_{1}, s_{2} \in S$ such that $s_{1}-s_{2} \notin S$ and $s_{2}-s_{1} \notin S$. Then $\left(s_{1}, s_{2}\right)+\left(s_{1}, s_{2}\right)^{-1} \subsetneq S$.

The second proof of (2.4). By (3. 5) we have $M^{*}=\bigcap_{\Pi}\left(M+S_{P}\right)$.

(3. 6). Assume that $S$ is integrally closed. Let $\Pi$ be the set of maximal $t$-ideals. Assume that $A^{t}=\bigcap_{\Pi}\left(A+S_{P}\right)$ for each $A \in f(S)$. Then $S$ is a PVMS.

PROOF. Let $P \in \Pi$. Let $a, b \in S$. We have $a+b \in(2 a, 2 b)^{v}$. Then $a+b \in(2 a, 2 b)+S_{P}$. By [13, Lemma 11], $(a, b)+S_{P}$ is principal. By [13, Lemma 13], $S_{P}$ is a valuation semigroup. By (3. 5), $S$ is a PVMS. and

(3. 7) PROPOSITION. $S$ is a PVMS if and only if $S$ is integrally closed

$$
\left(A_{1} \cap \cdots \cap A_{n}\right)^{t}=\left(A_{1}\right)^{t} \cap \cdots \cap\left(A_{n}\right)^{t}
$$

for all $A_{i} \in F(S)$.

PROOF. The necessity follows from (3. 5). The sufficiency. By the similar proof of $(2) \Longrightarrow(1)$ of $\left[1\right.$, Theorem 4], it follows that $A^{t}=\bigcap_{\Pi}\left(A+S_{P}\right)$ for each $A \in \mathrm{F}(S)$. (3. 6) implies that $S$ is a PVMS. 
(3. 8) ([2, Proposition 6. 5], [11, Theorem (11. 4) (1)]). $D[X ; S]$ is a PVMD if and only if $S$ is a PVMS and $D$ is a $P V M D$.

(3. 9) (A PART of [1, Theorem 6]). Let $D$ be an integrally closed domain. Then $D$ is a $P V M D$ if and only if for $A_{i} \in F(D)$.

$$
\left(A_{1} \cap \cdots \cap A_{n}\right)^{t}=\left(A_{1}\right)^{t} \cap \cdots \cap\left(A_{n}\right)^{t}
$$

(3. 7), (3. 8) and (3. 9) imply the following,

(3. 10) THEOREM. Assume that $D$ is integrally closed and $S$ is integrally closed. Then the following conditions are equivalent.

for $A_{i} \in F(D[X ; S])$.

(1) $\left(A_{1} \cap \cdots \cap A_{n}\right)^{t}=\left(A_{1}\right)^{t} \cap \cdots \cap\left(A_{n}\right)^{t}$

(2) $\left(A_{1} \cap \cdots \cap A_{n}\right)^{t}=\left(A_{1}\right)^{t} \cap \cdots \cap\left(A_{n}\right)^{t}$

for $A_{i} \in F(D)$ and

$\left(A_{1} \cap \cdots \cap A_{n}\right)^{t}=\left(A_{1}\right)^{t} \cap \cdots \cap\left(A_{n}\right)^{t}$

for $A_{i} \in F(S)$.

The second proof of the necessity of (3. 7). By (3. 8), $k[X ; S]$ is a PVMD. Let $A_{1}, \cdots, A_{n} \in \mathrm{F}(S)$. By (3. 9), we have It follows that

$$
\left(A_{1} k[X ; S] \cap \cdots \cap A_{n} k[X ; S]\right)^{t}=\left(A_{1} k[X ; S]\right)^{t} \cap \cdots \cap\left(A_{n} k[X ; S]\right)^{t} .
$$

$$
\left(A_{1} \cap \cdots \cap A_{n}\right)^{t}=\left(A_{1}\right)^{t} \cap \cdots \cap\left(A_{n}\right)^{t} .
$$

The third proof of the necessity of (3. 7). Then we have $\left(A+A^{-1}\right)^{t}=S$. Hence we may apply (2. 4) for the t-operation.

The similar proof with [1, Corollary 4. 2] shows the following,

(3. 11). Let * be a star-operation on $S$ of finite character. Let $\Pi$ be the set of maximal *-ideals of $S$. Then the following conditions are equivalent.

(1) $\left(A_{1} \cap \cdots \cap A_{n}\right)^{*}=\left(A_{1}\right)^{*} \cap \cdots \cap\left(A_{n}\right)^{*}$ for each $A_{i} \in F(S)$.

(2) $A^{*}=\cap_{\mathrm{II}}\left(A+S_{P}\right)$

for each $A \in F(S)$.

(3) $(A: S B)^{*}=A^{*}: S B^{*}$ for $A \in F(S)$ and $B \in f(S)$.

§4. In this section we show that the conjecture stated at the begining of this paper is true for some examples.

If each ideal of $S$ is finitely generated, then $S$ is called Noetherian semigroup. A semigroup $S^{\prime}$ such that $S \subset S^{\prime} \subset G=\mathrm{q}(S)$ is called oversemigroup of $S$. Let $S^{\prime}$ be an oversemigroup of $S$. If $S^{\prime}$ satisfies

$$
(A \cap B)+S^{\prime}=\left(A+S^{\prime}\right) \cap\left(B+S^{\prime}\right)
$$

for each $A, B \in \mathrm{F}(S)$, then $S^{\prime}$ is called f-oversemigroup. If $S$ satisfies the ascending chain condition for integral v-ideals, then $S$ is called Mori semigroup.

Replacing "flat overring" by "f-oversemigroup", we have the following, 
(4. 1) PROPOSITION. [1, Theorem 1, Theorem 2, Theorem 3, Corollary 3.1, Theorem 4, Corollary 4.1, Corollary 4.2, Corollary 4.3 and Theorem 6] hold for $S$.

Let $I, J \in \mathrm{F}(S)$. If $I^{v} \subset J^{v}$, we set $I^{v} \geq J^{v}$. If $S$ is the intersection of a set of essential valuation oversemigroups with finite character, then we say that $S$ is of Krull type. If $S$ is the intersection of the set of essential valuation oversemigroups, then $S$ is called essential semigroup.

Replacing "Prüfer ring" by "valuation semigroup", we have the following,

(4. 2) PROPOSITION. [6, Theorem 1, Proposition 2, Proposition 3, Proposition 4, Theorem 5, Corollary to Theorem 5, Theorem 7, Proposition 10, Corollary to Proposition 10, Lemma 11 and Proposition 12] hold for S.

We call $S$ a crescent semigroup if, for any collection $\left\{A_{\lambda} \mid \lambda\right\} \subset \mathrm{f}(S)$ with $\cap_{\lambda} A_{\lambda} \neq \phi$ we have $\left(\cap_{\lambda} A_{\lambda}\right)^{v}=\cap_{\lambda}\left(A_{\lambda}\right)^{v}$.

(4. 3) PROPOSITION. [3, Theorem 1, Theorem 2, Theorem 3, Theorem 4 and Theorem 7] hold for $S$.

A prime ideal $P$ of $S$ is called strongly prime if $x, y \in G$ and $x+y \in P$ imply that $x \in P$ or $y \in P$. $S$ is called pseudo-valuation semigroup if every prime ideal of $S$ is strongly prime.

(4. 4) PROPOSITION. [7, Proposition 1.1, Proposition 1.2, Corollary 1.3, Theorewm 1.4, Lemma 1.6, Theorem 1.7, Proposition 2.4, Corollary 2.5, Proposition 2.6, Theorem 2.7, Proposition 2.8, Corollary 2.9, Theorem 2.10, Proposition 2.11, Lemma 2.12, Theorem 2.13, Proposition 2.14, Corollary 2.15 and Theorem 3.1] hold for $S$.

By a part of (4. 4), we have the following,

(4. 5) (The Semigroup Version of [3, Proposition 8]). Let $S$ be a pseudo-valuation semigroup which is not a valuation semigroup with the maximal ideal $M$ and valuation semigroup $V=M^{-1}$. Suppose that

(a) the ideals of $V$ are all of the form $x+V$ or $x+M$ for $x \in V$,

(b) $V$ is not a union of a finite number of principal fractional ideals of $S$.

Then, for all $A \in f(S)$ and $B \in F(S)$ we have $\left(A: B^{-1}\right)^{v}=(A+B)^{v}$.

(4. 6) (The SEMigroup Version of [6, Proposition 8]). Let $\Omega$ be a family of valuations defining $S$. Let $G^{\prime}$ be a torsion-free abelian group with a subgroup $G=q(S)$ and integral over $G$. Let $\Omega^{\prime}$ be the family of all the extensions of the valuations in $\Omega$ to $G^{\prime}$. Then

(a) $\Omega^{\prime}$ defines the integral closure $S^{\prime}$ of $S$ in $G^{\prime}$. 
(b) all extensions of essential valuations for $S$ are essential for $\boldsymbol{S}^{\prime}$. character.

(c) if $\Omega$ is of finite character and $\left(G^{\prime}: G\right)<\infty$, then $\Omega^{\prime}$ is of finite

(4. 7). [6, Corollaries to Proposition 8] hold for S.

We have proved the semigroup versions of [6].

(4. 8) (A SEMIGRoup VERSION OF [7, ThEOREM 1.5]). The following conditions are equivalent.

(a) $S$ is a pseudo-valuation semigroup.

(b) For each $x \in G-S$ and for each nonunit a of $S$, we have $a-x \in S$.

If $I^{v}$ is principal for each $I \in \mathrm{f}(S)$, then $S$ is called pseudo-Bezout semigroup or GCD-semigroup. We see that [14, Lemma, Theorem 1] hold for $S$. Therefore we have,

(4. 9). [7, Proposition 2. 2] holds for $S$.

Let $s_{1}, \cdots, s_{n}$ be a sequence of elements of $S$. If

(1) $\left(s_{1}, \cdots, s_{n}\right) \underset{\neq}{ } S$

(2) $\left(s_{1}, \cdots, s_{i}\right): s s_{i+1}=\left(s_{1}, \cdots, s_{i}\right) \quad(1 \leq i \leq n-1)$,

then $s_{1}, \cdots, s_{n}$ is called a regular sequence of $S$. If $S$ is the intersection of a family of discrete valuation semigroups of $\mathrm{q}(S)$ with finite character, then $S$ is called Krull semigroup.

(4. 10) (The Semigroup Version of [3, Theorem 5]).

(1) Assume that $S$ is not a group. Let $S$ be a crescent semigroup that is also a Mori semigroup. Then the maximal ideal $M$ is the unique maximal $v$-ideal of $S$. If $S$ is a Noetherian crescent semigroup, then the length of every regular sequence of $S$ is less than 2.

(2) Let $S$ be a Krull semigroup that is not a group. Then, $S$ is a crescent semigroup if and only if $S$ is a discrete valuation semigroup.

We have proved the semigroup versions of [3].

(4. 11). [9, Proposition 1.1] holds for $S$.

The maximal number $n$ such that there exists a chain $P_{1} \supsetneqq P_{2} \supsetneqq \ldots$ $\underset{\supsetneq}{\supset} P_{n}$ of prime ideals of $S$ is called dimension of $S$. Minimal prime ideals of $S$ are also called height one prime ideals. 
(4. 12) (The Semigroup version of [1, Theorem 5]). Let $S$ be a Mori semigroup which is not a group. Then, for $S$.

(a) The equivalence of (1),(2),(3),(4),(5) and (8) of [1, Theorem 5] holds

(b) The following statements are equivalent.

( $\alpha) A^{v}=\cap\left\{A+S_{P} \mid P \in v-M a x(S)\right\}$ for each $A \in F(S)$.

( $\beta$ ) For each maximal $v$-ideal $P$ of $S, S_{P}$ is one-dimensional and Noetherian in which every ideal is a v-ideal.

$(\gamma)$ For each height one prime ideal $P$ of $S$, every ideal of $S_{P}$ is a $v$-ideal and $S=\cap\left\{S_{P} \mid h t(P)=1\right\}$.

PROOF. Assume $(\alpha)$. Let $P$ be a maximal v-ideal. Then $S_{P}$ is Noetherian. By (4.11), every ideal of $S_{P}$ is a v-ideal. By [15], $S_{P}$ is one dimensional. Next assume $(\beta)$. Let $P$ be a height one prime ideal of $S$. Choose $x \in P$. Let $T=\left\{I \in \mathrm{F}(S) \mid x \in I \subset I^{v} \subset P\right\}$.

Then $T$ contains a maximal member $Q$. Assume $a+b \in P$, where $a \notin Q$ and $b \notin Q$. We have $(Q, a)^{v} \not \subset P$ and $(Q, b)^{v} \not \subset P$. Hence

$P \not \supset\left((Q, a)^{v}+(Q, b)^{v}\right)^{v}=((Q, a)+(Q, b))^{v} \subset Q^{v} \subset P$

a contradiction. We proved that $Q$ is a prime ideal of $S$. Hence $P=Q$ and $P$ is a maximal v-ideal.

As a semigroup version of $[1$, Corollary 5.1$]$, we have,

(4. 13). Assume that $S$ is Noetherian and integrally closed. Then ( $A \cap$ $B)^{v}=A^{v} \cap B^{v}$ and $(A: S B)^{v}=\left(A^{v}: s B^{v}\right)$ for all $A, B \in F(S)$.

PROOF. By [4, Section 2], $S$ is a Krull semigroup. Hence $S_{P}$ is a discrete valuation semigroup for every height one prime ideal $P$ of $S$.

(4. 14). [1, Theorem 7] holds for $S$.

PROOF. The necessity. By a part of (4.1) it suffices to show that the maximal ideal $M$ of $S$ is a $*$-ideal. Suppose that $M$ is not a $*$-ideal. Then $M^{*}=S$. Since $*$ has finite character, there is a finitely generated ideal $I \subset M$ with $I^{*}=S$. Let $x \in M$. Let

$$
T=\left\{I \in \mathrm{F}(S) \mid x \in I \subset I^{*} \subset M\right\} \text {. }
$$

Then $T$ contains a maximal element $P$. Then $P=P^{*}$. Assume that $a+b \in P$, where $a \in M-P$ and $b \in M-P$. We have $(P, a)^{*}=(P, b)^{*}=S$. Hence

$$
S=\left((P, a)^{*}+(P, b)^{*}\right)^{*}=((P, a)+(P, b))^{*} \subset P^{*} \subset M
$$

a contradiction. We proved that $P$ is a prime ideal of $S$. The rest is similar with that of $[1$, Theorem 7$]$. 
(4. 15) (The semigroup version of [1, Corollary 7. 1]). Assume that $S$ is a Mori semigroup which is not a group. Then, if $S$ is a crescent semigroup, then $S$ is one-dimensional and every element of $F(S)$ is a v-ideal.

We have proved the semigroup versions of [1].

(4. 16). [7, Proposition 3. 2] holds for S.

PROOF. We confer the semigroup versions of $[7$, Theorem 2. 7] and its proof. $S$ has a valuation oversemigroup $V$ with maximal ideal $M$. Since $M$ is a finitely generated ideal of $V, V$ is a discrete valuation semigroup. On the other hand, every prime ideal of $S$ is also a prime ideal of $V$. Therefore $\operatorname{dim}(S) \leq 1$.

(4. 17). [8, Lemma 2. 2] holds for $S$.

Replacing '(4) $V$ is a two-generated $R$-module' as '(4) $V$ is a twogenerated fractional ideal of $S$, we have,

(4, 18). [7, Theorem 3. 5] holds for $S$.

For the proof we use (4. 17).

(4. 19). [7, Corollary 3. 3] holds for $S$.

PROOF. Let $S$ be a Noetherian pseudo-valuation semigroup which is not a group. Let $M$ be the maximal ideal of $S$. By the proof of (4. 16), $M$ is a minimal prime ideal of $S$, and there exists a discrete valuation oversemigroup $V$ with maximal ideal $M$. Let $v$ be the valuation of $V$. Let $T$ be an oversemigroup of $S$ and let $P$ be a prime ideal of $T$. There exists a valuation oversemigroup $W$ of $T$ such that $Q \cap T=P$ for some prime ideal $Q$ of $W$. Let $w$ be the valuation of $W$. Let $y \notin V$. Since $v(y)<0$, we have $-y \in M$. Hence $w(-y)>0$ and $y \notin W$. Therefore $W \subset V$. Then $M$ is a prime ideal of $W$. Since $M$ is a finitely generated ideal of $S$, we have $M=x+W$ for some $x \in M$. Then we have $M=x+V$. It follows $W=V$. Hence $Q=M$ and $P=M$. Namely $M$ is the only prime ideal of $T$. Hence $T$ is a pseudo-valuation semigroup.

(4. 20). [7, Corollary 3. 4] holds for S.

PROOF. Let $S, M, V$ and $v$ be as in the proof of (4.19). Let $S^{\prime}$ be the integral closure of $S$. The proof of (4.19) shows that $V$ is the only non-trivial valuation oversemigroup of $S$. By [5, Theorem 12. 8], we have $S^{\prime}=V$. 
We have proved the semigroup versions of [7].

We know some conditions for $D[X ; S]$ to have a.c.c.p. property (cf. [11, Section 9]). As an appendix, we will note the followings. Assume that $S$ is an additive subsemigroup of the nonnegative rational numbers. For a domain $D$, $D[X ; S]$ has a.c.c.p. if and only if $D$ has a.c.c.p. and $S$ has a.c.c.p. Let $n$ be a natural number and $s \in S$. If the equation $n x=s$ has a solution in $S$, then we say that $n$ divides $s$. Assume that $S$ has a.c.c.p. Then, for each nonzero $s \in S$ and for each prime number $p$, the natural numbers $n$ such that $p^{n}$ divide $s$ are of finite number. Moreover there exists an example $S$ which satisfies this condition and does not have a.c.c.p. For example, set $S=\left\{\frac{2^{n}+a}{5^{n}} \mid 1 \leq n, 0 \leq a\right\} \cup\{0\}$.

The author wishes to express his acknowledgement to his teacher Mr. Iwao Okada for encouragement given him during writing this paper.

\section{References}

[1] D.D. Anderson, Star-operations induced by overrings, Communications in Alg. 16 (1988), 2535-2553.

[2] D.D. Anderson and D.F. Anderson, Divisorial ideals and invertible ideals in a graded integral domain, J. Alg. 76 (1982), 549-569.

[3] D.D. Anderson, D.F. Anderson, D.L. Costa, D.E. Dobbs, J.L. Mott and M. Zafrullah, Some characterizations of v-domains and related properties, Colloquium Math. 58 (1989), 1-9.

[4] L. Chouinard, Krull semigroups and divisor class groups, Canad. J. Math. 33 (1981), 1459-1468.

[5] R. Gilmer, Commutative Semigroup Rings, The Univ, of Chicago Press, Chicago, 1984.

[6] M. Griffin, Some results on v-multiplication rings, Canad. J. Math. 19 (1967), 710-722.

[7] J. Hedstrom and E. Houston, Pseudo-valuation domains, Pacific J. Math. 75 (1978), 137-147.

[8] W. Heinzer, Integral domains in which each nonzero ideal is divisorial, Mathematika 15 (1968), 164-170.

[9] E. Houston, T. Lucas and T. Viswanathan, Primary decomposition of divisorial ideals in Mori domains, J. Alg. 117 (1988), 327-342.

[10] R. Matsuda, Notes on Prüfer v-multiplication rings, Bull. Fac. Sci., Ibaraki Univ. 12 (1980), 9-15.

[11] R. Matsuda, Torsion-free abelian semigroup rings VI, Bull. Fac. Sci., Ibaraki Univ. 18 (1986), 23-43.

[12] R. Matsuda and A. Okabe, On an AACDMZ question (A characterization of v-domains), Proc. 14-th Sympos on Commutative Ring Theory (1992), 137-141.

[13] R. Matsuda and K. Satô, Kronecker function rings of semigroups, Bull. Fac. Sci., Ibaraki Univ. 19 (1987), 31-46.

[14] S. McAdam, Two conductor theorems, J. Alg. 23 (1972), 239-240.

[15] K. Satô and R. Matsuda, On M-semigroups, Memoirs Tohoku Inst. Tech. 8 (1988), 1-7. 